\title{
Mutations in DNA damage response pathways as a potential biomarker for immune checkpoint blockade efficacy: evidence from a seven-cancer immunotherapy cohort
}

\author{
Wenjing Zhang ${ }^{1,}{ }^{*}$, Liwen Zhang ${ }^{1,}{ }^{*}$, Hao Jiang ${ }^{1}$, Yuting $\mathrm{Li}^{2}$, Suzhen Wang ${ }^{1}$, Qinghua Wang ${ }^{1}$ \\ ${ }^{1}$ Department of Health Statistics, Key Laboratory of Medicine and Health of Shandong Province, School of Public \\ Health, Weifang Medical University, Weifang, Shandong 261053, China \\ ${ }^{2}$ Tianjin Cancer Institute, National Clinical Research Center for Cancer, Key Laboratory of Cancer Prevention and \\ Therapy of Tianjin, Tianjin Medical University Cancer Institute and Hospital, Tianjin 300060, China \\ *Equal contribution
}

Correspondence to: Qinghua Wang, Suzhen Wang; email: wangqinghua wfmc@163.com, https://orcid.org/0000-0003-39123174; wangsz@wfmc.edu.cn

Keywords: immunotherapy, DDR mutations, predictive biomarker, microenvironment, clinical practice

Received: July 3, 2021

Accepted: October 26, 2021

Published: November 8, 2021

Copyright: (c) 2021 Zhang et al. This is an open access article distributed under the terms of the Creative Commons Attribution License (CC BY 3.0), which permits unrestricted use, distribution, and reproduction in any medium, provided the original author and source are credited.

\section{ABSTRACT}

Recently several studies have demonstrated the implications of mutations in DNA damage response (DDR) pathways for immune checkpoint blockade (ICB) treatment. However, smaller sample sizes, lesser cancer types, and the lack of multivariate-adjusted analyses may produce unreliable results. From the Memorial SloanKettering Cancer Center (MSKCC) cohort, we curated 1363 ICB-treated patients to evaluate the association of DDR mutations with immunotherapy prognosis. Besides, 4286 ICB-treated-naive patients from the Cancer Genome Atlas (TCGA) cohort were used to explore the intrinsic prognosis of DDR mutations. Factors in the microenvironment regarding DDR mutations were also assessed. We found that patients with DDR mutations exhibited a significantly prolonged immunotherapy overall survival via multivariate Cox model in the MSKCC cohort (HR: 0.70, $P<0.001$ ). Specific cancer analyses revealed that patients with DDR mutations could obtain the better ICB prognosis in bladder cancer and colorectal cancer (HR: $0.59[P=0.034]$ and $0.33[P=0.006]$ ). Stratified analyses showed that age $>60$, male gender, high mutation burden, and PD-1/PD-L1 treatment were the positive conditions for ICB survival benefits of DDR mutations (all $P<0.01$ ). Mutations of 4 DDR genes, including MRE11A, MSH2, ATM, and POLE could predict favorable ICB prognoses (all $P<0.01$ ). A better immune microenvironment was observed in DDR mutated patients. Mutations in DDR pathways or single DDR genes were associated with preferable ICB efficacy in specific cancers or subpopulations. Findings from our study would provide clues for tailing clinical trials and immunotherapy strategies.

\section{INTRODUCTION}

Immune checkpoint blockade (ICB) therapies dramatically extended the survival interval of advanced tumors, however, the durable response was only observed in a subset of patients [1, 2]. Efficacy of ICB treatment could be predicted by multiple biomarkers, such as expression of programmed death receptor 1 ligand (PD-L1) [3, 4], tumor mutation burden (TMB) [5], neoantigen burden (NB) [6], mRNA expression signatures [7], and gut microbiome [8]. Their effectiveness would sometimes be lost in specific settings and each biomarker has a limiting application.

Difficulties in determining cut-off values, biases of distinct test platforms, and dynamic changes have 
reduced the broad utilization of PD-L1 expression [7]. Elevated TMB and NB were reported to be correlated with ICB treatment efficacy [5, 9], however, no uniform cut-off values were determined to select patients with a high mutational burden in distinct tumors [7]. Mismatch repair deficiency (dMMR) is another FDA-approved vital indicator owing to its ability to increase TMB and NB $[1,10]$. However, less than $5 \%$ of tumor patients are dMMR-related, this reality may be a restrained factor for the extensive application of dMMR [11].

Six DNA damage response (DDR) signals (i.e., mismatch repair [MMR], nucleotide excision repair [NER], homologous recombination [HR], Fanconi anemia [FA], checkpoints, and specific DDR genes) are mainly existed to perform the function of genome maintenance, and thus preserve the genomic integrity [12]. Causally, alterations in any pathway or gene of DDR would induce the subtype with elevated TMB and MB [13]. Mutations of the MMR pathway were clinically correlated with durable ICB response $[1,14]$. Mehnert et al. reported that endometrial cancer patients with POLE mutations exhibited favorable pembrolizumab efficacy [14]. Hugo et al. found that BRCA2-mutant melanoma patients harbored better clinical benefits of anti-PD-1 therapy [6]. Recently another study demonstrated that co-mutations of DDR signals were remarkably associated with elevated mutational load, increased immune signatures enrichment, better objective response rate, and prolonged ICB survival [15]. However, the limited sample size and cancer type of the above studies may influence the produced results. Besides, the predictive abilities of mutations of most DDR genes are poorly studied in clinical immunotherapy.

Herein, we curated a seven-cancer cohort to explore the association of DDR mutations with ICB efficacy in distinct subgroups. In addition, mutations of single DDR gene association with ICB clinical benefit were also evaluated. As a comparison, patients without ICB treatment from the Cancer Genome Atlas (TCGA) were used to assess the intrinsic prognostic ability of DDR mutations. Results from this study may give more implications for tailoring clinical ICB therapy.

\section{RESULTS}

\section{Clinical characteristics and DDR mutations of included patients}

Among 1363 ICB-treated tumor patients, 115 (8.4\%) with BG, $211(15.5 \%)$ with BLCA, 109 (8.0\%) with CRC, 129 (9.5\%) with HNSC, 344 (25.2\%) with NSCLC, $142(10.4 \%)$ with RCC, and 313 (23.0\%) with SKCM. Overall, 1083 (79.5\%) were treated with
anti-PD-1/PD-L1 therapy, 76 (5.6\%) were anti-CTLA-4 therapy, and $204(14.9 \%)$ were combined therapy. The median ICB survival interval was 12 months. Other detailed clinical information was curated in Supplementary Table 1.

Overall, $493(36.2 \%)$ tumors harbored mutations in at least one DDR gene and $870(63.8 \%)$ tumors were the DDR wild-type subgroup. The mutational patterns of 34 DDR genes were illustrated in Figure 1.

\section{Association of DDR mutations with prognosis in ICB-treated and ICB-treated-naive patients}

In the MSKCC cohort contained ICB-treated patients, survival analysis showed that patients with DDR mutations exhibited a significantly better overall survival (OS) than patients without DDR mutations (median OS: 34 [95\% CI, 27-47] vs. 16 [95\% CI, 1419] months; Log-rank test, $P<0.001$; Figure $2 \mathrm{~A})$. To obtain a more accurate association, we conducted a multivariate Cox regression model with confounding variables (i.e., age, gender, cancer subtype, drug target, and TMB) taken into consideration. The result was still statistically significant (HR: $0.70,95 \%$ CI: $0.58-0.85$, $P<0.001$; Figure 2B).

In the TCGA cohort, univariate survival analysis produced a consistent result as compared with the MSKCC cohort, that is DDR-mutated patients were significantly correlated with better prognosis (median OS: 67.4 [95\% CI, 61.1-87.3] vs. 57.7 [95\% CI, 53.363.8] months; Log-rank test, $P=0.007$; Figure 2C). We also observed the significant association in multivariate Cox model after adjusting clinical confounders (HR: $0.85,95 \%$ CI: $0.75-0.96, P=0.008$; Figure 2D).

DDR mutations were linked with favorable outcomes in both cohorts, however, DDR-mutated patients had more survival benefits in the MSKCC cohort than in the TCGA cohort (HR: 0.70 vs. 0.85; Figure 2B, 2D). These results indicate more positive roles of DDR mutations for predicting prognosis in immunotherapy settings.

\section{DDR mutations association with prognosis in 7 distinct cancers}

We evaluated the prognostic ability of DDR mutations in 7 distinct cancer subtypes with MSKCC and TCGA cohorts. In this section, multivariate Cox models with confounders adjusted were performed only when survival curves were statistically significant.

Univariate analysis in the MSKCC cohort showed that DDR mutations were significantly associated with 
favorable outcomes in 4 cancers, including BLCA, CRC, NSCLC, and SKCM (all log-rank test $P<0.05$; Figure 3B, 3C, 3E, 3G). After controlling the confounding factors, only BLCA and CRC exhibited the survival benefits of DDR-mutated patients (HR: BLCA $[0.59,95 \% \mathrm{CI}, 0.36-0.96, P=0.034]$, CRC $[0.33,95 \%$ CI, 0.15-0.75, $P=0.008]$; Figure 3B, 3C). DDR mutations in NSCLC and SKCM showed trends of better prognosis, however, they did not reach the statistical significance in multivariate analysis (HR: NSCLC [0.76, 95\% CI, 0.55-1.05, $P=0.098]$, SKCM [0.68, 95\% CI, 0.42-1.10, $P=0.117]$; Figure 3E, 3G). We further used the TCGA data to calculate the intrinsic prognostic ability of DDR mutations in BLCA and CRC. Multivariate Cox model was not significant when controlling relevant confounders in BLCA (HR: 0.82 , 95\% CI: $0.59-1.15, P=0.251$; Figure $3 \mathrm{I}$ ), and
DDR-mutated CRC patients were also not related to the prognosis (Log-rank test $P=0.752$; Figure $3 \mathrm{~J}$ ). The above results suggest the potential prediction implications of DDR mutations for immunotherapy efficacy in patients with BLCA and CRC.

SKCM patients with DDR mutations did not exhibit the survival benefits in the MSKCC cohort. However, in the non-ICB-treated TCGA cohort, DDR-mutated SKCM patients harbored a significantly better survival outcome than wild-type patients (HR: $0.53,95 \%$ CI: $0.38-0.75$, $P<0.001 ;$ Figure $3 \mathrm{~N})$.

DDR mutations were not correlated with patients' survival in BG, HNSC, NSCLC, and RCC in both MSKCC and TCGA cohorts (all Log-rank test $P>0.05$; Figure 3A, 3D, 3F, 3H, 3K, 3L, 3M).

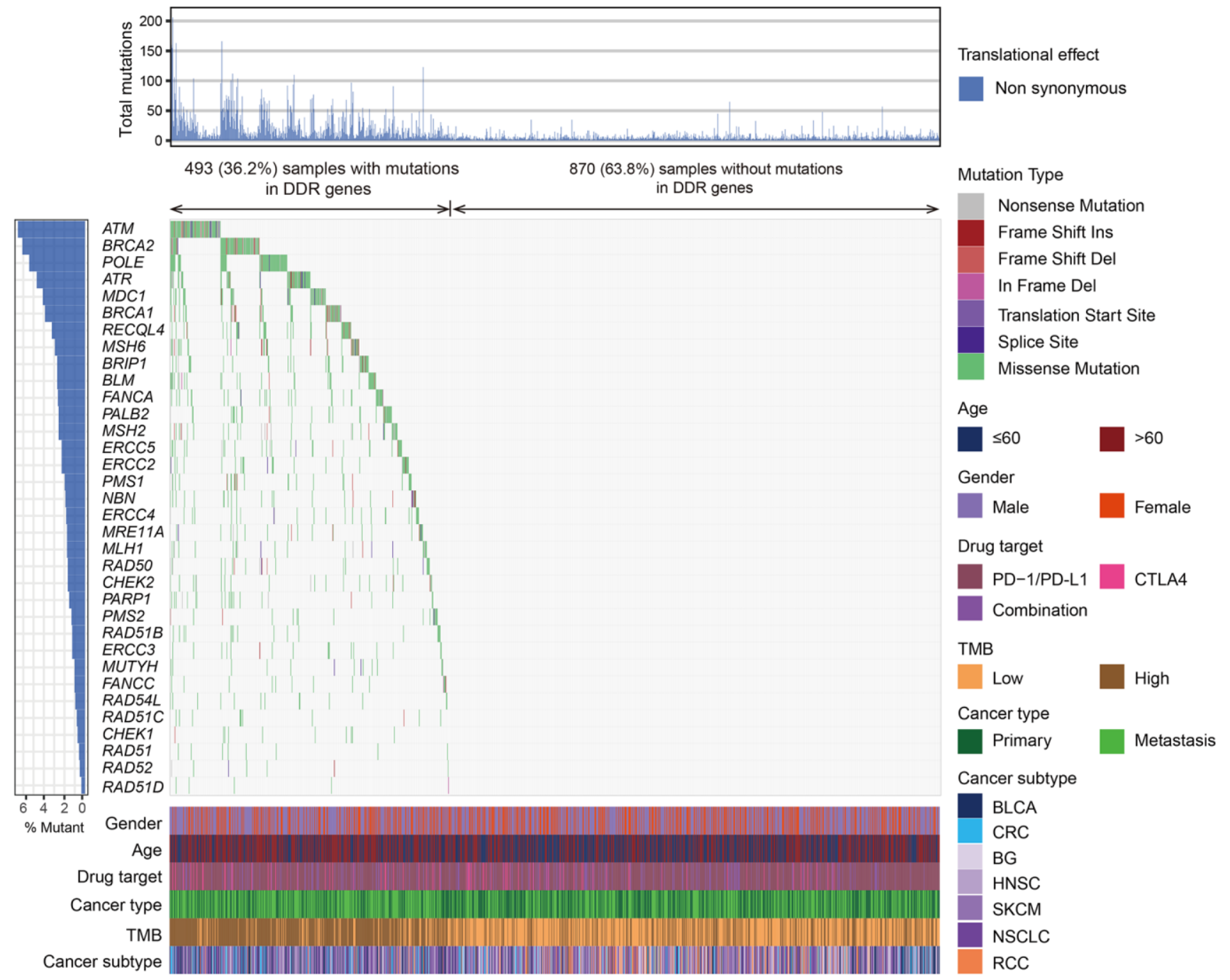

Figure 1. The mutational pattern of 34 DDR genes among 1363 patients treated with ICB agents. The left panel represents gene mutation rates, the upper panel indicates the non-synonymous mutation counts of each patient, the middle panel shows mutational landscape of all DDR genes with distinct mutation types color coded distinctly, and the bottom panel displays clinical characteristics such as age, gender, drug target, TMB, and cancer subtype. 


\section{DDR mutations versus prognosis in distinct clinical conditions}

To illuminate whether the immunotherapy and prognostic implications of DDR mutations were influenced by specific clinical factors, we performed stratified analysis and multivariate Cox regression model of DDR mutations in distinct subpopulations with MSKCC and TCGA data.

For patients with age $\leq 60$, no significant correlation was observed between DDR mutations and prognosis in MSKCC $(P=0.198)$, however, DDR mutations could

A

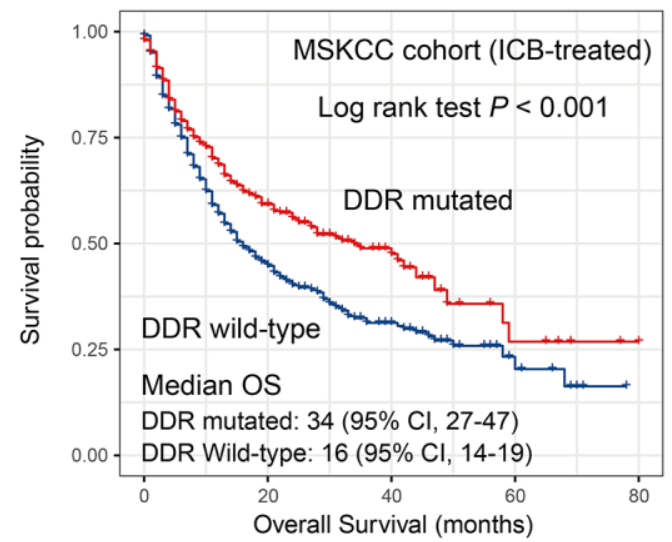

Number at risk

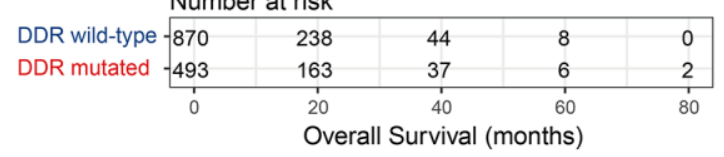

B

\begin{tabular}{|c|c|c|c|c|}
\hline Variable & $\mathbf{N}$ & HR & HR (95\% Cl) & $P$ \\
\hline \multicolumn{5}{|l|}{ Age } \\
\hline$\leq 60$ & 555 & $\phi$ & Reference & \\
\hline$>60$ & 808 & $a$ & $0.92(0.78,1.08)$ & 0.30 \\
\hline \multicolumn{5}{|l|}{ Gender } \\
\hline Female & 496 & $\phi$ & Reference & \\
\hline Male & 867 & 9 & $0.93(0.79,1.10)$ & 0.40 \\
\hline \multicolumn{2}{|l|}{ Cancer subtype } & 1 & & \\
\hline BG & 115 & - & Reference & \\
\hline BLCA & 211 & $\rightarrow 1$ & $0.82(0.60,1.12)$ & 0.22 \\
\hline CRC & 109 & $\longrightarrow 1$ & $0.73(0.50,1.06)$ & 0.09 \\
\hline HNSC & 129 & 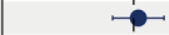 & $1.06(0.77,1.46)$ & 0.72 \\
\hline NSCLC & 344 & $\rightarrow$ & $0.99(0.76,1.30)$ & 0.95 \\
\hline RCC & 142 & $\bullet$ & $0.32(0.22,0.45)$ & $<0.001$ \\
\hline SKCM & 313 & 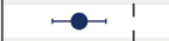 & $0.51(0.36,0.71)$ & $<0.001$ \\
\hline \multicolumn{5}{|l|}{ Drug target } \\
\hline PD-1/PD-L1 & 1083 & ๑ & Reference & \\
\hline CTLA-4 & 76 & $\bullet-1$ & $0.64(0.42,0.98)$ & 0.04 \\
\hline Combination & 204 & $\rightarrow 1$ & $0.62(0.47,0.80)$ & $<0.001$ \\
\hline \multicolumn{5}{|l|}{ TMB } \\
\hline Low & 681 & $\varphi$ & Reference & \\
\hline High & 682 & - & $0.92(0.77,1.10)$ & 0.35 \\
\hline \multicolumn{2}{|l|}{ DDR status } & 1 & & \\
\hline Wild type & 870 & 0 & Reference & \\
\hline Mutated & 493 & -1 & $0.70(0.58,0.85)$ & $<0.001$ \\
\hline
\end{tabular}

predict favorable survival in TCGA $(P=0.002)$. DDRmutated patients with age $>60$ harbored a better prognosis in MSKCC $(P<0.001)$, this result was not significant in TCGA $(P=0.268)$ (Figure 4; Supplementary Table 2).

Both male and female patients exhibited the survival benefits of DDR mutations in the ICB-treated cohort $(P=0.003$ and 0.044$)$. In the further analysis in TCGA, DDR-mutated male patients were not predictive of prognosis $(P=0.069)$, but female patients with DDR mutations obtained a more significant result $(P=0.003)$ (Figure 4; Supplementary Table 2).
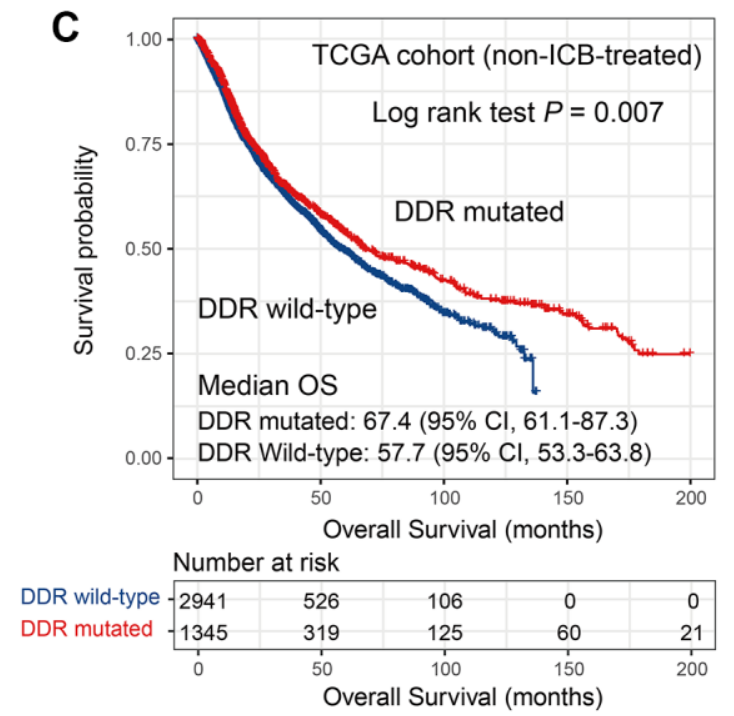

D

\begin{tabular}{|c|c|c|c|c|c|}
\hline \multirow{2}{*}{\multicolumn{6}{|c|}{$\begin{array}{l}\text { Variable } \\
\text { Age }\end{array}$}} \\
\hline & & & & & \\
\hline$\leq 60$ & 1951 & & 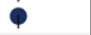 & Reference & \\
\hline$>60$ & 2335 & & $1 \bullet$ & $1.78(1.59,1.99)$ & $<0.001$ \\
\hline \multicolumn{6}{|l|}{ Gender } \\
\hline Female & 1609 & & $\varphi$ & Reference & \\
\hline Male & 2677 & & - & $1.04(0.93,1.15)$ & 0.504 \\
\hline Stage & & & 1 & & \\
\hline$|-| \mid$ & 2464 & & $\phi$ & Reference & \\
\hline III-IV & 1822 & & 1 & $2.92(2.62,3.26)$ & $<0.001$ \\
\hline Cancer subtype & & & i & & \\
\hline$B G$ & 893 & & b & Reference & \\
\hline BLCA & 407 & $\bullet$ & 1 & $0.45(0.37,0.56)$ & $<0.001$ \\
\hline CRC & 512 & - & 1 & $0.24(0.19,0.31)$ & $<0.001$ \\
\hline HNSC & 438 & $\bullet$ & 1 & $0.39(0.32,0.47)$ & $<0.001$ \\
\hline NSCLC & 953 & $\bullet$ & 1 & $0.59(0.49,0.71)$ & $<0.001$ \\
\hline $\mathrm{RCC}$ & 679 & - & 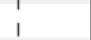 & $0.22(0.18,0.27)$ & $<0.001$ \\
\hline SKCM & 404 & - & i & $0.38(0.31,0.47)$ & $<0.001$ \\
\hline TMB & & & 1 & & \\
\hline Low & 2143 & & b & Reference & \\
\hline High & 2143 & & 甲 & $1.03(0.89,1.18)$ & 0.716 \\
\hline DDR status & & & 1 & & \\
\hline Wild type & 2941 & & $\phi$ & Reference & \\
\hline Mutated & 1345 & & $a^{\prime}$ & $0.85(0.75,0.96)$ & 0.008 \\
\hline
\end{tabular}

Figure 2. Association of DDR mutations with survival outcome in MSKCC and TCGA cohorts. (A-B) DDR mutations versus survival outcome with univariate analysis and multivariate regression model in the MSKCC cohort; (C-D) DDR mutations versus survival outcome with univariate analysis and multivariate regression model in the TCGA cohort. 
DDR mutations could not predict prognosis in the lowTMB subgroup of both cohorts (both $P>0.05$ ). However, patients with high TMB and DDR mutations harbored improved survival times in both cohorts $(P<$ 0.001 and $P=0.007$ ), and the result was more significant in the MSKCC cohort than in the TCGA cohort (HR: 0.56 vs. 0.83) (Figure 4; Supplementary Table 2).

Patients from MSKCC who received distinct immunotherapies also exhibited inconsistent association between DDR mutations and survival outcomes. DDR

\section{MSKCC cohort (ICB-treated)}
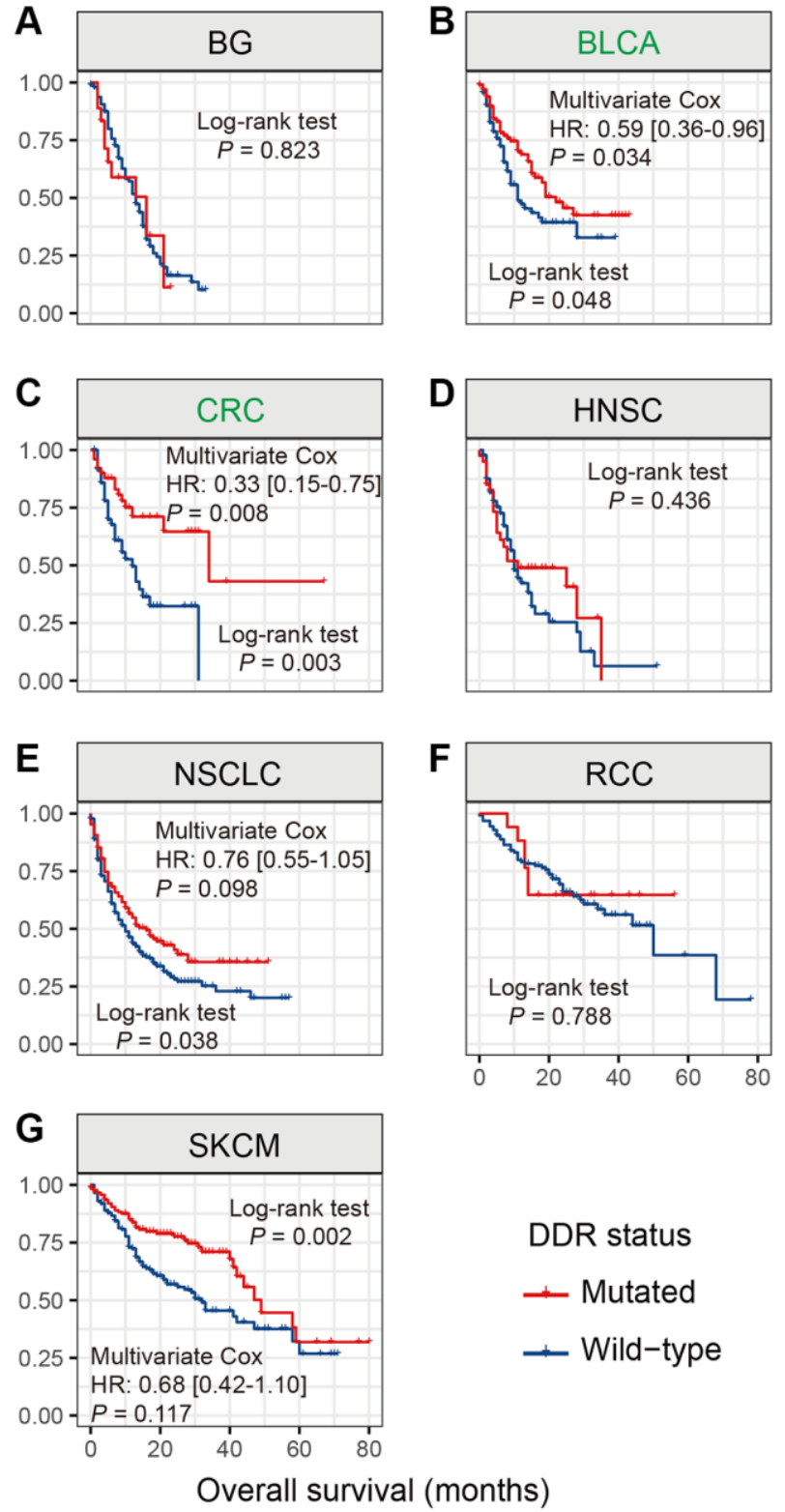

mutations were positively correlated with immunotherapy prognosis in patients treated with PD-1/ PD-L1 agents $(P=0.002)$, rather than patients treated with CTLA-4 agents or combined therapy (both $P>$ 0.05) (Figure 4; Supplementary Table 2).

\section{Mutations of single DDR gene versus prognosis}

To understand the implications of each DDR gene for immunotherapy efficacy and prognosis, we evaluated the association of mutations in single DDR genes with patients' survival in 2 cohorts.

\section{TCGA cohort (non-ICB-treated)}
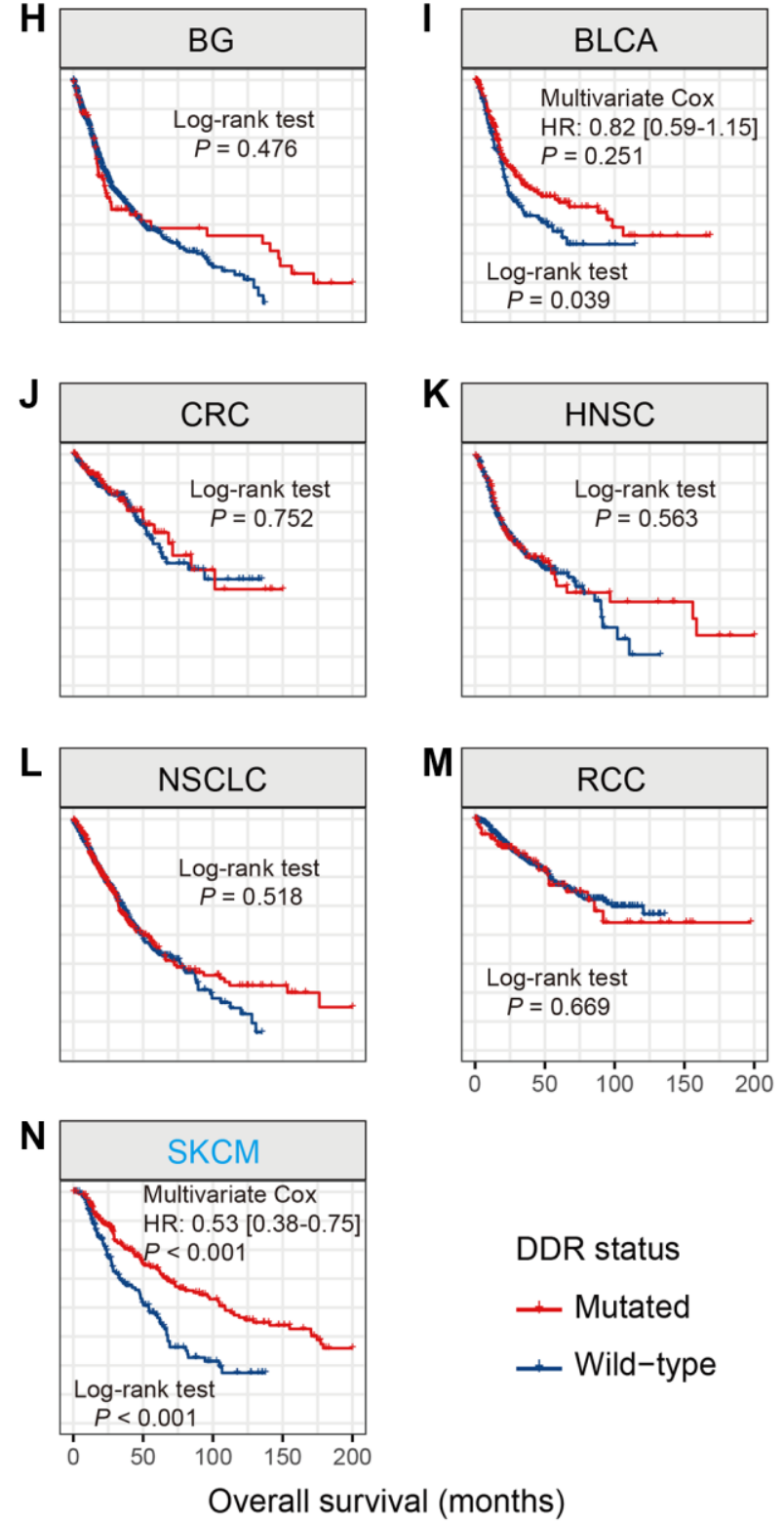

Figure 3. DDR mutations association with prognosis in 7 distinct cancers. (A-G) Association of DDR mutations with prognosis in 7 cancers in the MSKCC cohort; $(\mathbf{H}-\mathbf{N})$ Association of DDR mutations with prognosis in 7 cancers in the TCGA cohort. Cancer types that exhibited the survival benefits of NLRP3 mutations in the MSKCC and TCGA cohorts were respectively colored with green and blue. 
Univariate survival analysis in the MSKCC cohort showed that 8 DDR genes (i.e., MSH2, MRE11A, NBN, BRCA2, RAD51C, ATM, POLE, and PARP1) mutations were associated with the preferable survival outcome (all Log-rank $P<0.05$; Supplementary Table 3). Multivariate Cox regression model was conducted with the mutations of identified 8 DDR genes and clinical confounding factors (i.e., age, gender, cancer type, drug target, and $\mathrm{TMB}$ ) taken into consideration. Results showed that 2 genes, including ATM and MRE11A, still exhibited the positive association with prognosis (both multivariate-adjusted $P<0.05$; Figure 5, Supplementary Figure 1). And these 2 genes mutations association with prognosis in TCGA were not statistically significant (both $P>0.05$; Supplementary Table 3).

Besides, in the TCGA cohort, Kaplan-Meier survival analysis revealed that 3 DDR genes (i.e., MSH6, $B R C A 1$, and $A T R$ ) mutations were related to the survival outcome (all Log-rank $P<0.05$;
Supplementary Figure 2). And this association remained still significant even adjusted for confounding variables (all multivariate-adjusted $P<0.05$; Supplementary Figure 3). Mutations in BRCAl or ART were correlated with better survival outcomes, however, MSH6 mutations were predictive of worse prognosis.

\section{Factors in the microenvironment concerning DDR mutations}

We explored the correlation between microenvironment factors and DDR mutations to explain why DDRmutated patients harbored better immunotherapy prognosis.

Tumor infiltration CD8 $\mathrm{T}$ cells were significantly enriched in the patients with DDR mutations $(P<$ 0.001; Figure 6A). Macrophages M1 and M2 play immune-promotion and immune-suppressive roles, respectively. We observed that DDR-mutated patients

\section{Mutated DDR vs. Prognosis}

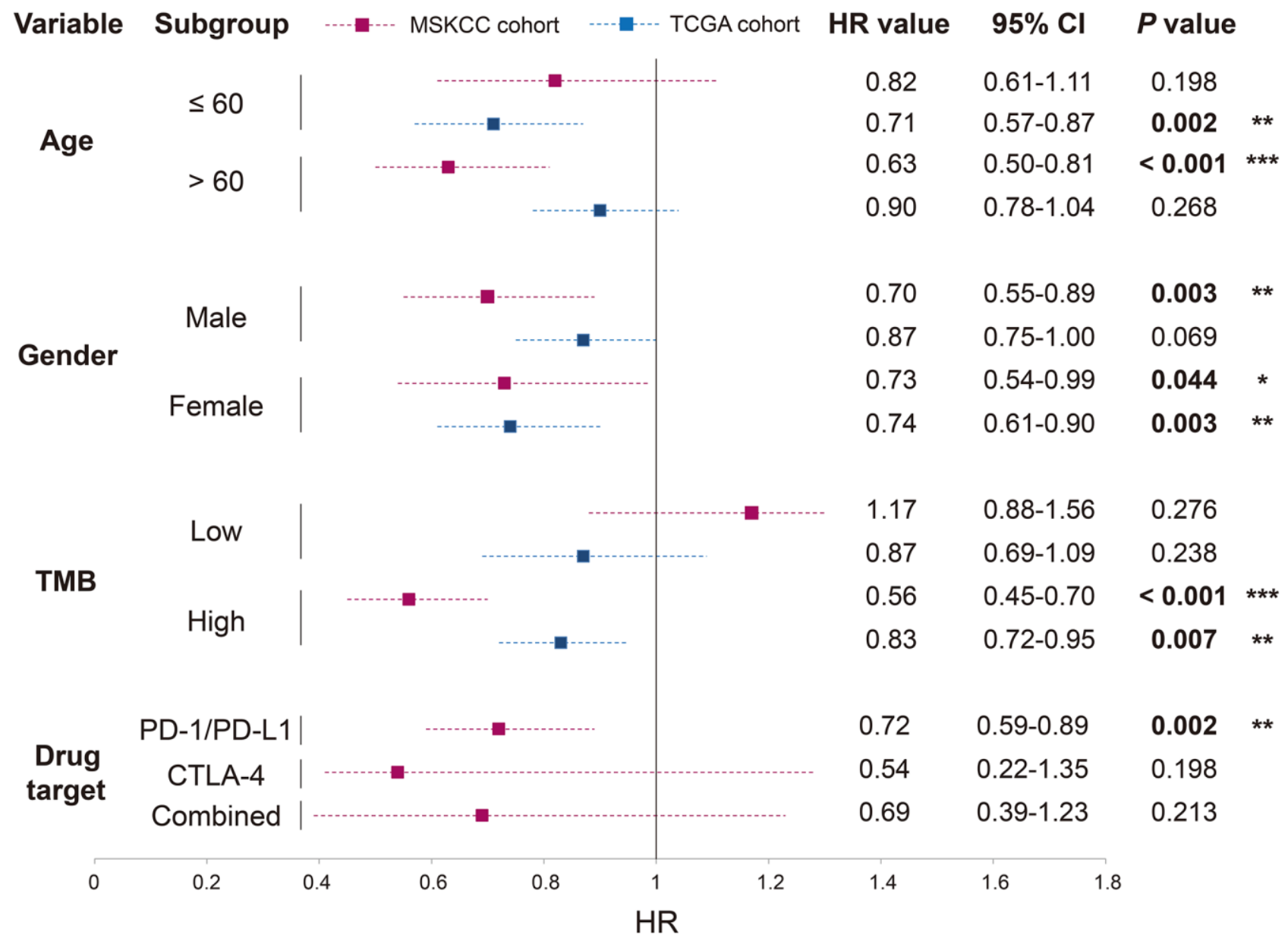

Figure 4. Association of DDR mutations with survival outcome in distinct clinical settings based on the data from MSKCC and TCGA cohorts. HR value, $95 \% \mathrm{Cl}$, and $P$ value were derived from multivariate Cox regression model with clinical factors adjusted. ${ }^{*} P<0.05 ;{ }^{* *} P<0.01 ;{ }^{* * *} P<0.001$. 
had elevated infiltration of M1 macrophages and decreased infiltration of M2 macrophages (both $P<$ 0.001; Figure 6A). Besides, patients with DDR mutations also harbored the elevated infiltration of resting and activated memory $\mathrm{CD} 4 \mathrm{~T}$ cells, $\mathrm{T}$ follicular helper cells, monocytes, and activated dendritic cells (all $P<0.001$; Figure 6A).

Among 33 immune checkpoints, 27 (81.8\%) were significantly up-regulated in DDR-mutated patients, such as CD274, PDCD1, and CTLA4 (all $P<0.001$; Figure $6 \mathrm{~B}$ ). These results further verify the potential implications of DDR mutations for predicting ICB efficacy.

Of the 15 curated immune-related signatures, 12 $(80.0 \%)$ were highly enriched in mutated DDR patients (all $P<0.001$; Figure 6C). Especially, enrichment of IFN- $\gamma$ signature and $\mathrm{T}$ cell-flamed signature, which were previously reported to be predictive of better ICB prognosis, were observed in DDR-mutated patients (both $P<0.001$; Figure 6C).

\section{DISCUSSION}

Previously several studies have reported the roles of DDR mutations in immunotherapy [12, 15]. However, smaller sample sizes, lesser cancer types, and lack of multivariate-adjusted analysis may introduce some biases into the generated results. In this study, by using an aggregated ICB cohort with 7 cancers, we performed multi-dimension analyses between DDR mutations and ICB treatment prognosis. As a comparison, non-ICB-

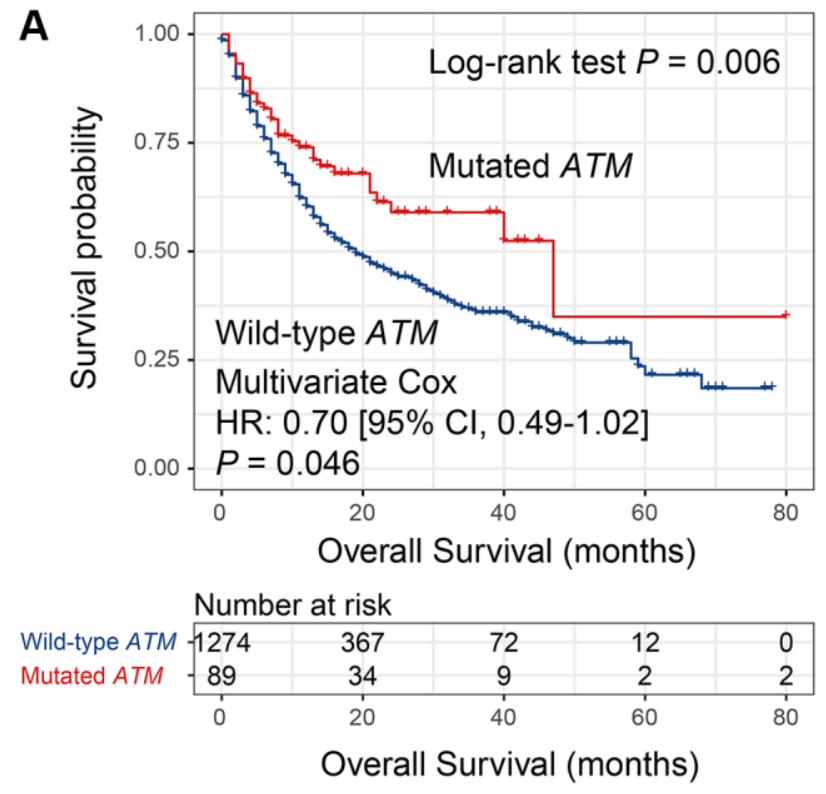

treated patients from the TCGA cohort were also used to determine the potential ICB prediction and intrinsic prognosis abilities of DDR mutations. Several novel discoveries of our study would provide clues for tailing immunotherapeutic strategies.

Patients with DDR mutations exhibited more survival benefits in the MSKCC cohort as compared with the TCGA cohort. DDR pathways play vital roles in maintaining genome integrity; and mutations in DDR signals could produce the acceleration of genomic alterations (i.e., TMB). Previous many studies have demonstrated that a high TMB was linked with the preferable immunotherapy efficacy [16-20]. This may be a reason for explaining the more survival benefits of DDR mutated patients in the MSKCC. In addition, the increased infiltration of immuneresponse cells, the decreased infiltration of immunesuppressive cells, and elevated enrichment of numerous immune-related signatures were markedly enriched in patients with DDR mutations. These immune relevant factors to some extent contribute to the better prognosis of DDR mutated patients; however, they could play larger roles in the settings with immunotherapy. In a word, the more survival benefits of DDR mutations in the MSKCC cohort may be associated with the elevated TMB and favorable immune microenvironment.

DNA is continually exposed to the endogenous and exogenous damages, and the coordinated activity of multiple DDR pathways is needed to maintain genomic integrity under normal cellular conditions [21, 22].

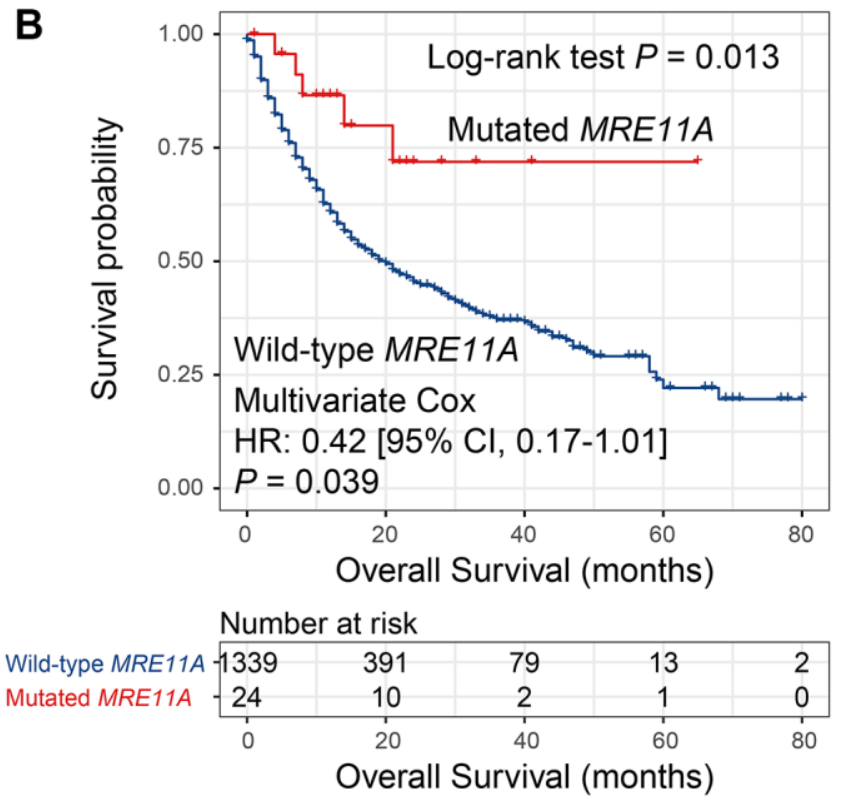

Figure 5. Univariate and multivariate Cox regression analyses of mutations in 2 DDR genes in the MSKCC cohort. Survival curves representation of mutations in (A) ATM and (B) MRE11A. 
Mutations in DDR signals could result in the failure to repair DNA damage and induce a variety of genomic alterations [10]. In some cases, changes produced by these genomic aberrations may serve as the antigens to the immune system and thus drive tumor initiation and immunogenicity [23, 24]. Acceleration of the genomic changes may be recognized as the neoantigens by the immune system, and push it to release more immune activity factors. The open microenvironment couples with immunotherapy could markedly enhance the treatment effects. In this study, DDR mutated patients harbored the preferable ICB survival outcomes, which may be implicated in the elevated TMB and neoantigen burden.
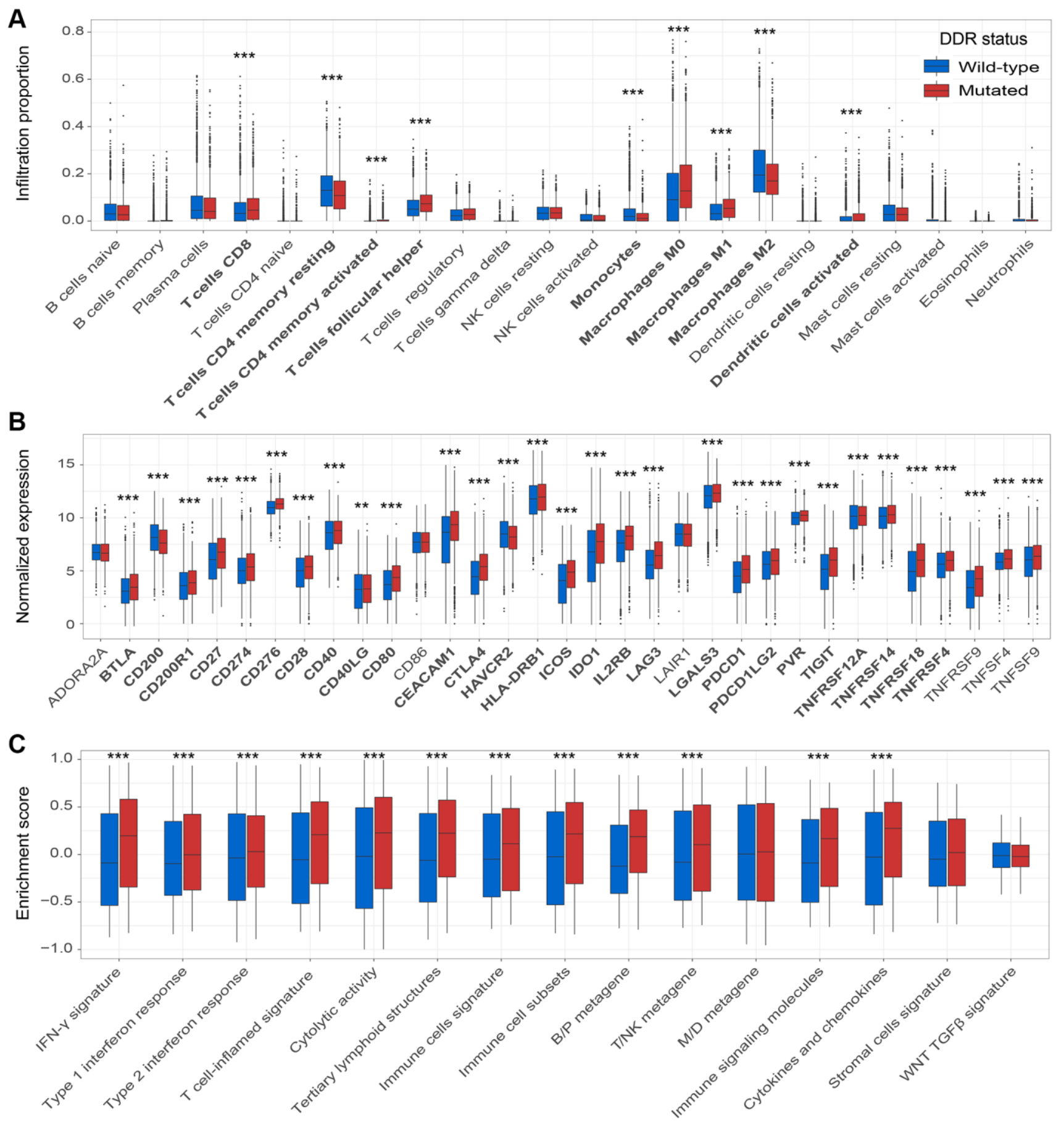

Figure 6. DDR mutations association with factors in the immune microenvironment. (A) Diverse infiltration abundance of 22 immune cells based on DDR mutational status; (B) Distinct expression of 33 immune checkpoints in patients with and without DDR mutations; (C) Distinct enrichment of 15 immune signatures in patients with and without DDR mutations. ${ }^{*} P<0.05$; ${ }^{* *} P<0.01$; ${ }^{* * *} P<0.001$. 
In specific cancer analysis, DDR mutations were correlated with favorable ICB survival in BLCA and CRC patients, while this correlation was not found in the other 5 cancers (i.e., BG, HNSC, NSCLC, RCC, and SKCM). Wang et al. used the integrated genomic and immunotherapy data and reported that the ICB treatment prognosis of patients with DDR mutations was superior to that of those without DDR mutations in NSCLC and SKCM [15]. The inconsistent results may attribute to the smaller sample size Wang et al. used as compared with our study (sample size: NSCLC (34 vs. 344), SKCM (174 vs. 313)). Although the significant association of DDR mutations with favorable ICB survival was not observed in SKCM patients received immunotherapy, in non-ICB-treated SKCM patients, DDR mutations were markedly correlated with better survival outcome, indicating that DDR mutations may play a more important role in predicting SKCM intrinsic prognosis rather than ICB prognosis. The firstly comprehensive analysis between DDR mutations and prognosis across multiple cancers demonstrated that BLCA or CRC patients with DDR mutations may obtain a prolonged survival interval in immunotherapy settings.

Previous studies have pointed out the age and sex differences in immune response and immunotherapy efficacy $[25,26]$. In our study, younger (age $\leq 60)$ and female patients with DDR mutations did not exhibit the ICB treatment benefits. Inversely, older (age > 60) and male patients with DDR mutations harbored a remarkably better ICB prognosis. Consistent with our results, a recent study reported that younger and female patients always obtain a poorer response in clinical studies, this phenomenon may be correlated to the more poorly presented drive mutations these patients accumulated [27]. DDR-mutated patients with a high TMB also exhibit a favorable ICB prognosis. The high $\mathrm{TMB}$, which is a stimulating factor for the activation of lymphocyte $\mathrm{T}$ cells, may provide a suitable environment for immunotherapy. Noticeably, in patients received anti-PD-1/PD-L1 agents, DDR mutations were linked with preferable survival outcome. However, this result was not observed in patients who received anti-CTLA-4 agents or combined therapy. By analyzing the association of DDR mutations with ICB efficacy in distinct clinical settings, we found that age $>60$, male gender, high TMB, and anti-PD-1/PD-L1 treatment are the positive factors for the immunotherapy prognosis of DDR mutations.

Instead of choosing $\mathrm{TMB}$ or $\mathrm{NB}$ as predictors for immunotherapy efficacy, mutations in single genes, such as POLE [28], POLD1 [28], PBRM1 [29], MUC16 [30, 31], and TTN [32] could also obtain the equivalent effects. In this study, mutations in 2 DDR genes (i.e., ATM and MRE11A) were associated with better ICB survival. Among, the positive link between ATM mutations and ICB benefits in BLCA was recently demonstrated [33], and this link was further verified based on 7 cancers in our study. Besides, MRE11A mutations correlation with improved survival was also firstly discovered in this study. These 2 genes we reported may harbor vital implications for evaluating immune checkpoint-based therapy efficacy.

We finally explored the links between factors in the microenvironment and DDR mutations. Immune cells represented by $\mathrm{CD} 8 \mathrm{~T}$ cells infiltration, immune checkpoints (e.g., CD274, PDCD1, and CTLA4) expression, and immune-related signatures (e.g., IFN- $\gamma$ and $\mathrm{T}$ cell-inflamed signatures) were highly enriched in the patients with DDR mutations. The better immune microenvironment may be the explanation for the preferable ICB prognosis of DDR mutations.

By using the aggregated ICB cohort and performing multi-dimension analyses, we found that DDR mutations were associated with better ICB survival outcomes in pan-seven-cancers. Besides, in specific cancers (e.g., BLCA and CRC) and in distinct clinical settings (e.g., age > 60, male gender, high TMB, and anti-PD-1/PD-L1 treatment), patients with DDR mutations also exhibited the ICB survival benefits. Mutations in MSH2, MRE11A, ATM, and POLE were all correlated with the favorable ICB prognosis. Findings derived from our study would provide evidence and basics for guiding clinical immunotherapy.

\section{MATERIALS AND METHODS}

\section{Genomic data and clinical information of included patients}

Somatic mutation data and clinical information of 1363 patients treated with ICB therapy were collected from the Memorial Sloan-Kettering Cancer Center (MSKCC) [16]. Among, brain glioma (BG), bladder cancer (BLCA), colorectal cancer (CRC), head and neck cancer (HNSC), non-small cell lung cancer (NSCLC), renal cell carcinoma (RCC), and melanoma (SKCM) were included for related analyses. Detailed clinical characteristics of these patients were curated in Supplementary Table 1.

Mutation and clinical data of 4286 patients contained above 7 cancers in TCGA were downloaded from Genome Data Commons (https://portal.gdc.cancer.gov). A total of 4021 patients with both gene expression and mutation data were used for immune microenvironment analysis. 


\section{DDR genes and determination of DDR mutations}

From a previous study reported by MSKCC [12], we collected 34 DDR-related genes of 6 pathways (Supplementary Table 4). Patients with nonsynonymous mutations (i.e., missense mutation, nonsense mutation, frameshift indel, inframe indel, splice site, and translation start site) of DDR genes were considered to be DDR-mutated.

\section{DDR mutations versus tumor-immune microenvironment}

Based on the gene expression data from the TCGA cohort, we calculated and evaluated the enrichment of 3 factors in the microenvironment (i.e., tumor infiltration immune cells, immune checkpoint, and immune-related signatures) according to DDR mutation status.

Tumor infiltration immune cells proportion was calculated with the CIBERSORT algorithm, which is a useful tool to estimate the abundances of 22 immune cell types with gene expression data [34].

Ye et al. integrated a list of 34 immune checkpoint genes [35], in this study, the gene of VSIR was not found in the mRNA expression profile. Therefore, the expression of 33 immune checkpoints was analyzed.

Immune signatures that represented distinct immunological and cellular features were aggregated as follows: 1) Interferon- $\gamma$ (IFN- $\gamma$ ) signature, which exhibits vital roles in activation and promotion of anti-tumor immune response, and it was reported to be associated with immunotherapy clinical benefits [36]; 2) T cell-inflamed signature, which is consisted of 18 immune genes correlated with dendritic and CD8 T cells activity [36]; 3) cytolytic activity, which reflects the activity of cytotoxic $\mathrm{T}$ cells and its released cytolytic factors to kill tumor cells [37]; 4) tertiary lymphoid structures (TLS), which are ectopic lymphoid organs related to cancer prognosis, immunity response, and ICB therapy efficacy [38]; 5) immune and stromal cells signature, which indicates the proportion of immune and stromal cells in mixed tumor tissue [39]; 6) immune cell subsets, which means the abundance of $\mathrm{T}$ cells, $\mathrm{B}$ cells, and natural killer (NK) cells [40]; 7) B/P, T/NK, and M/D metagenes were reported to be correlated with the enrichment of B cells/plasma cells, T cells/NK cells, and monocytes/dendritic cells, respectively [41]; 8) immune signaling molecules [40]; 9) cytokines and chemokines [40]; and 10) WNT TGF $\beta$ signature, which plays the suppression roles in immune response [42].

\section{Gene set variation and enrichment analysis}

Enrichment scores of the abovementioned immune signatures were assessed with single sample gene set enrichment analysis (ssGSEA) method from GSVA package (V1.36.1) [43] according to the expression values of each gene in signatures. Differential analysis of TCGA sequencing data was performed with DESeq2 package (V1.28.1) [44]. Gene set enrichment analysis (GSEA) embedded in fgsea package (V1.14.0) (https://github.com/ctlab/fgsea) was utilized to calculate pathways enriched in distinct subgroups. Pathways from the Kyoto Encyclopedia of Genes and Genomes (KEGG) were used as the background dataset.

\section{Statistical analyses}

$\mathrm{R}$ software (V4.0.1) and its packages were used to performed relevant analyses. The mutational pattern exhibited in the waterfall plot was drawn via GenVisR package (V1.20.0) [45]. Kaplan-Meier approach and Log-rank test were used to generate survival curves and to compare the difference significance of two curves, separately. Through forestmodel package (V0.5.0), multivariate Cox regression models were performed to adjust confounding factors and to produce forest plots. For the association of continuous variables with DDR mutation status, Wilcoxon rank-sum test was utilized. $P$ values less than 0.05 were considered to be statistically significant unless a particular specification.

\section{AUTHOR CONTRIBUTIONS}

QW and SW designed this study; QW, WZ, LZ, and YL developed the methodology and acquired the related data; WZ, LZ, and YL performed data analysis and interpretation; WZ, QW, and $\mathrm{LZ}$ drafted and revised the manuscript; QW and SW supervised this study. All authors read and approved the final manuscript.

\section{ACKNOWLEDGMENTS}

Qinghua Wang thank Prof. Suzhen Wang at the Weifang Medical University for providing help in work and research.

\section{CONFLICTS OF INTEREST}

The authors declare no conflicts of interest related to this study.

\section{FUNDING}

This study was supported by the Shandong Provincial Youth Innovation Team Development Plan of Colleges and Universities (No. 2019-6-156, Lu-Jiao), National 
Natural Science Foundation of China (No. 81872719 and 81803337), Provincial Natural Science Foundation of Shandong Province (No. ZR201807090257), and National Bureau of Statistics Foundation Project (No. 2018LY79).

\section{REFERENCES}

1. Lemery S, Keegan P, Pazdur R. First FDA Approval Agnostic of Cancer Site - When a Biomarker Defines the Indication. N Engl J Med. 2017; 377:1409-12. https://doi.org/10.1056/NEJMp1709968 PMID:29020592

2. Larkin J, Chiarion-Sileni V, Gonzalez R, Grob JJ, Cowey $C L$, Lao $C D$, Schadendorf $D$, Dummer R, Smylie $M$, Rutkowski P, Ferrucci PF, Hill A, Wagstaff J, et al. Combined Nivolumab and Ipilimumab or Monotherapy in Untreated Melanoma. N Engl J Med. 2015; 373:23-34. https://doi.org/10.1056/NEJMoa1504030 PMID:26027431

3. Reck $M$, Rodríguez-Abreu $D$, Robinson $A G$, Hui $R$, Csőszi T, Fülöp A, Gottfried M, Peled N, Tafreshi A, Cuffe S, O'Brien M, Rao S, Hotta $K$, et al, and KEYNOTE-024 Investigators. Pembrolizumab versus Chemotherapy for PD-L1-Positive Non-Small-Cell Lung Cancer. N Engl J Med. 2016; 375:1823-33. https://doi.org/10.1056/nejmoa1606774 PMID:27718847

4. Herbst RS, Baas $P$, Kim DW, Felip E, Pérez-Gracia JL, Han JY, Molina J, Kim JH, Arvis CD, Ahn MJ, Majem M, Fidler MJ, de Castro G Jr, et al. Pembrolizumab versus docetaxel for previously treated, PD-L1-positive, advanced non-small-cell lung cancer (KEYNOTE-010): a randomised controlled trial. Lancet. 2016; 387:1540-50.

https://doi.org/10.1016/S0140-6736(15)01281-7 PMID:26712084

5. Carbone DP, Reck M, Paz-Ares L, Creelan B, Horn L, Steins M, Felip E, van den Heuvel MM, Ciuleanu TE, Badin F, Ready N, Hiltermann TJN, Nair S, et al, and CheckMate 026 Investigators. First-Line Nivolumab in Stage IV or Recurrent Non-Small-Cell Lung Cancer. N Engl J Med. 2017; 376:2415-26.

https://doi.org/10.1056/NEJMoa1613493 PMID:28636851

6. Hugo W, Zaretsky JM, Sun L, Song C, Moreno BH, HuLieskovan S, Berent-Maoz B, Pang J, Chmielowski B, Cherry G, Seja E, Lomeli S, Kong X, et al. Genomic and Transcriptomic Features of Response to Anti-PD-1 Therapy in Metastatic Melanoma. Cell. 2016; 165:35-44. https://doi.org/10.1016/i.cell.2016.02.065 PMID:26997480
7. Gibney GT, Weiner LM, Atkins MB. Predictive biomarkers for checkpoint inhibitor-based immunotherapy. Lancet Oncol. 2016; 17:e542-51. https://doi.org/10.1016/S1470-2045(16)30406-5 PMID:27924752

8. Gopalakrishnan V, Helmink BA, Spencer CN, Reuben A, Wargo JA. The Influence of the Gut Microbiome on Cancer, Immunity, and Cancer Immunotherapy. Cancer Cell. 2018; 33:570-80. https://doi.org/10.1016/j.ccell.2018.03.015 PMID:29634945

9. Van Allen EM, Miao D, Schilling B, Shukla SA, Blank C, Zimmer L, Sucker A, Hillen $U$, Foppen MHG, Goldinger SM, Utikal J, Hassel JC, Weide B, et al. Genomic correlates of response to CTLA-4 blockade in metastatic melanoma. Science. 2015; 350:207-11.

https://doi.org/10.1126/science.aad0095 PMID:26359337

10. Mouw KW, Goldberg MS, Konstantinopoulos PA, D'Andrea AD. DNA Damage and Repair Biomarkers of Immunotherapy Response. Cancer Discov. 2017; 7:675-93.

https://doi.org/10.1158/2159-8290.CD-17-0226 PMID:28630051

11. Cortes-Ciriano I, Lee S, Park WY, Kim TM, Park PJ. A molecular portrait of microsatellite instability across multiple cancers. Nat Commun. 2017; 8:15180. https://doi.org/10.1038/ncomms15180 PMID:28585546

12. Teo MY, Seier K, Ostrovnaya I, Regazzi AM, Kania BE, Moran MM, Cipolla CK, Bluth MJ, Chaim J, AlAhmadie H, Snyder A, Carlo MI, Solit DB, et al. Alterations in DNA Damage Response and Repair Genes as Potential Marker of Clinical Benefit From PD-1/PD-L1 Blockade in Advanced Urothelial Cancers. J Clin Oncol. 2018; 36:1685-94. https://doi.org/10.1200/ico.2017.75.7740 PMID:29489427

13. Lee JK, Choi YL, Kwon M, Park PJ. Mechanisms and Consequences of Cancer Genome Instability: Lessons from Genome Sequencing Studies. Annu Rev Pathol. 2016; 11:283-312. https://doi.org/10.1146/annurev-pathol-012615$\underline{044446}$ PMID:26907526

14. Mehnert JM, Panda A, Zhong H, Hirshfield K, Damare S, Lane K, Sokol L, Stein MN, Rodriguez-Rodriquez L, Kaufman HL, Ali S, Ross JS, Pavlick DC, et al. Immune activation and response to pembrolizumab in POLEmutant endometrial cancer. J Clin Invest. 2016; 126:2334-40.

https://doi.org/10.1172/JCl84940

PMID:27159395 
15. Wang Z, Zhao J, Wang G, Zhang F, Zhang Z, Zhang F, Zhang Y, Dong H, Zhao X, Duan J, Bai H, Tian Y, Wan R, et al. Comutations in DNA Damage Response Pathways Serve as Potential Biomarkers for Immune Checkpoint Blockade. Cancer Res. 2018; 78:6486-96. https://doi.org/10.1158/0008-5472.can-18-1814 PMID:30171052

16. Samstein RM, Lee $\mathrm{CH}$, Shoushtari AN, Hellmann MD, Shen R, Janjigian YY, Barron DA, Zehir A, Jordan EJ, Omuro A, Kaley TJ, Kendall SM, Motzer RJ, et al. Tumor mutational load predicts survival after immunotherapy across multiple cancer types. Nat Genet. 2019; 51:202-6.

https://doi.org/10.1038/s41588-018-0312-8 PMID:30643254

17. Wang Z, Duan J, Cai S, Han M, Dong H, Zhao J, Zhu B, Wang S, Zhuo M, Sun J, Wang Q, Bai H, Han J, et al. Assessment of Blood Tumor Mutational Burden as a Potential Biomarker for Immunotherapy in Patients With Non-Small Cell Lung Cancer With Use of a NextGeneration Sequencing Cancer Gene Panel. JAMA Oncol. 2019; 5:696-702.

https://doi.org/10.1001/jamaoncol.2018.7098 PMID:30816954

18. Sholl LM, Hirsch FR, Hwang D, Botling J, Lopez-Rios F, Bubendorf L, Mino-Kenudson $M$, Roden AC, Beasley MB, Borczuk A, Brambilla E, Chen G, Chou TY, et al. The Promises and Challenges of Tumor Mutation Burden as an Immunotherapy Biomarker: A Perspective from the International Association for the Study of Lung Cancer Pathology Committee. J Thorac Oncol. 2020; 15:1409-24.

https://doi.org/10.1016/j.jtho.2020.05.019 PMID:32522712

19. Rizvi NA, Hellmann MD, Snyder A, Kvistborg $P$, Makarov V, Havel JJ, Lee W, Yuan J, Wong P, Ho TS, Miller ML, Rekhtman N, Moreira AL, et al. Cancer immunology. Mutational landscape determines sensitivity to PD-1 blockade in non-small cell lung cancer. Science. 2015; 348:124-8.

https://doi.org/10.1126/science.aaa1348

PMID:25765070

20. Hellmann MD, Callahan MK, Awad MM, Calvo E, Ascierto PA, Atmaca A, Rizvi NA, Hirsch FR, Selvaggi G, Szustakowski JD, Sasson A, Golhar R, Vitazka P, et al. Tumor Mutational Burden and Efficacy of Nivolumab Monotherapy and in Combination with Ipilimumab in Small-Cell Lung Cancer. Cancer Cell. 2018; 33:85361.e4.

https://doi.org/10.1016/i.ccell.2018.04.001 PMID:29731394

21. Hoeijmakers JH. DNA damage, aging, and cancer. N Engl J Med. 2009; 361:1475-85. https://doi.org/10.1056/nejmra0804615 PMID:19812404

22. Roos WP, Thomas AD, Kaina B. DNA damage and the balance between survival and death in cancer biology. Nat Rev Cancer. 2016; 16:20-33.

https://doi.org/10.1038/nrc.2015.2 PMID:26678314

23. Jeggo PA, Pearl LH, Carr AM. DNA repair, genome stability and cancer: a historical perspective. Nat Rev Cancer. 2016; 16:35-42.

https://doi.org/10.1038/nrc.2015.4 PMID:26667849

24. Bartkova J, Horejsí Z, Koed K, Krämer A, Tort F, Zieger K, Guldberg P, Sehested M, Nesland JM, Lukas C, $\emptyset$ rntoft T, Lukas J, Bartek J. DNA damage response as a candidate anti-cancer barrier in early human tumorigenesis. Nature. 2005; 434:864-70.

https://doi.org/10.1038/nature03482 PMID:15829956

25. Simon AK, Hollander GA, McMichael A. Evolution of the immune system in humans from infancy to old age. Proc Biol Sci. 2015; 282:20143085. https://doi.org/10.1098/rspb.2014.3085 PMID:26702035

26. Klein SL, Flanagan KL. Sex differences in immune responses. Nat Rev Immunol. 2016; 16:626-38. https://doi.org/10.1038/nri.2016.90 PMID:27546235

27. Castro A, Pyke RM, Zhang X, Thompson WK, Day CP, Alexandrov LB, Zanetti M, Carter $H$. Strength of immune selection in tumors varies with sex and age. Nat Commun. 2020; 11:4128. https://doi.org/10.1038/s41467-020-17981-0 PMID:32807809

28. Wang $F$, Zhao $Q$, Wang $Y N$, Jin $Y$, He MM, Liu ZX, $\mathrm{Xu} \mathrm{RH}$. Evaluation of POLE and POLD1 Mutations as Biomarkers for Immunotherapy Outcomes Across Multiple Cancer Types. JAMA Oncol. 2019; 5:1504-6. https://doi.org/10.1001/jamaoncol.2019.2963 PMID:31415061

29. Braun DA, Ishii Y, Walsh AM, Van Allen EM, Wu CJ, Shukla SA, Choueiri TK. Clinical Validation of PBRM1 Alterations as a Marker of Immune Checkpoint Inhibitor Response in Renal Cell Carcinoma. JAMA Oncol. 2019; 5:1631-3.

https://doi.org/10.1001/jamaoncol.2019.3158 PMID:31486842

30. Li X, Pasche B, Zhang W, Chen K. Association of MUC16 Mutation With Tumor Mutation Load and Outcomes in Patients With Gastric Cancer. JAMA Oncol. 2018; 4:1691-8. 
https://doi.org/10.1001/jamaoncol.2018.2805 PMID:30098163

31. Wang $Q$, Yang $Y$, Yang $M$, Li X, Chen K. High mutation load, immune-activated microenvironment, favorable outcome, and better immunotherapeutic efficacy in melanoma patients harboring MUC16/CA125 mutations. Aging (Albany NY). 2020; 12:10827-43. https://doi.org/10.18632/aging.103296 PMID:32491995

32. Jia $Q$, Wang J, He N, He J, Zhu B. Titin mutation associated with responsiveness to checkpoint blockades in solid tumors. JCl Insight. 2019; 4:e127901. https://doi.org/10.1172/ici.insight.127901 PMID:31092729

33. Yi R, Lin A, Cao M, Xu A, Luo P, Zhang J. ATM Mutations Benefit Bladder Cancer Patients Treated With Immune Checkpoint Inhibitors by Acting on the Tumor Immune Microenvironment. Front Genet. 2020; 11:933.

https://doi.org/10.3389/fgene.2020.00933 PMID: $\underline{32922441}$

34. Newman AM, Liu CL, Green MR, Gentles AJ, Feng W, Xu Y, Hoang CD, Diehn $M$, Alizadeh AA. Robust enumeration of cell subsets from tissue expression profiles. Nat Methods. 2015; 12:453-7. https://doi.org/10.1038/nmeth.3337 PMID:25822800

35. Ye $Y$, Jing $Y$, Li L, Mills GB, Diao L, Liu H, Han L. Sexassociated molecular differences for cancer immunotherapy. Nat Commun. 2020; 11:1779. https://doi.org/10.1038/s41467-020-15679-x PMID: $\underline{32286310}$

36. Ayers $M$, Lunceford J, Nebozhyn M, Murphy E, Loboda A, Kaufman DR, Albright A, Cheng JD, Kang SP, Shankaran V, Piha-Paul SA, Yearley J, Seiwert TY, et al. IFN- $\gamma$-related mRNA profile predicts clinical response to PD-1 blockade. J Clin Invest. 2017; 127:2930-40. https://doi.org/10.1172/JCl91190 PMID:28650338

37. Rooney MS, Shukla SA, Wu CJ, Getz G, Hacohen N. Molecular and genetic properties of tumors associated with local immune cytolytic activity. Cell. 2015; 160:48-61.

https://doi.org/10.1016/j.cell.2014.12.033

PMID:25594174

38. Finkin S, Yuan D, Stein I, Taniguchi K, Weber A, Unger $\mathrm{K}$, Browning JL, Goossens N, Nakagawa $\mathrm{S}$, Gunasekaran G, Schwartz ME, Kobayashi M, Kumada $H$, et al. Ectopic lymphoid structures function as microniches for tumor progenitor cells in hepatocellular carcinoma. Nat Immunol. 2015; 16:1235-44. https://doi.org/10.1038/ni.3290

PMID:26502405

39. Yoshihara K, Shahmoradgoli M, Martínez E, Vegesna R, Kim H, Torres-Garcia W, Treviño V, Shen H, Laird PW, Levine DA, Carter SL, Getz G, Stemke-Hale K, et al. Inferring tumour purity and stromal and immune cell admixture from expression data. Nat Commun. 2013; 4:2612.

https://doi.org/10.1038/ncomms3612

PMID:24113773

40. Cancer Genome Atlas Network. Genomic Classification of Cutaneous Melanoma. Cell. 2015; 161:1681-96.

https://doi.org/10.1016/j.cell.2015.05.044 PMID:26091043

41. Nagalla S, Chou JW, Willingham MC, Ruiz J, Vaughn JP, Dubey P, Lash TL, Hamilton-Dutoit SJ, Bergh J, Sotiriou C, Black MA, Miller LD. Interactions between immunity, proliferation and molecular subtype in breast cancer prognosis. Genome Biol. 2013; 14:R34. https://doi.org/10.1186/gb-2013-14-4-r34 PMID:23618380

42. Chen YP, Wang YQ, Lv JW, Li YQ, Chua MLK, Le QT, Lee $N$, Colevas $A D$, Seiwert T, Hayes DN, Riaz N, Vermorken JB, O'Sullivan B, et al. Identification and validation of novel microenvironment-based immune molecular subgroups of head and neck squamous cell carcinoma: implications for immunotherapy. Ann Oncol. 2019; 30:68-75.

https://doi.org/10.1093/annonc/mdy470

PMID:30407504

43. Hänzelmann S, Castelo R, Guinney J. GSVA: gene set variation analysis for microarray and RNA-seq data. BMC Bioinformatics. 2013; 14:7. https://doi.org/10.1186/1471-2105-14-7 PMID:23323831

44. Love MI, Huber W, Anders S. Moderated estimation of fold change and dispersion for RNA-seq data with DESeq2. Genome Biol. 2014; 15:550. https://doi.org/10.1186/s13059-014-0550-8 PMID:25516281

45. Skidmore ZL, Wagner AH, Lesurf R, Campbell KM, Kunisaki J, Griffith OL, Griffith M. GenVisR: Genomic Visualizations in R. Bioinformatics. 2016; 32:3012-4. https://doi.org/10.1093/bioinformatics/btw325 PMID:27288499 


\section{SUPPLEMENTARY MATERIALS}

\section{Supplementary Figures}

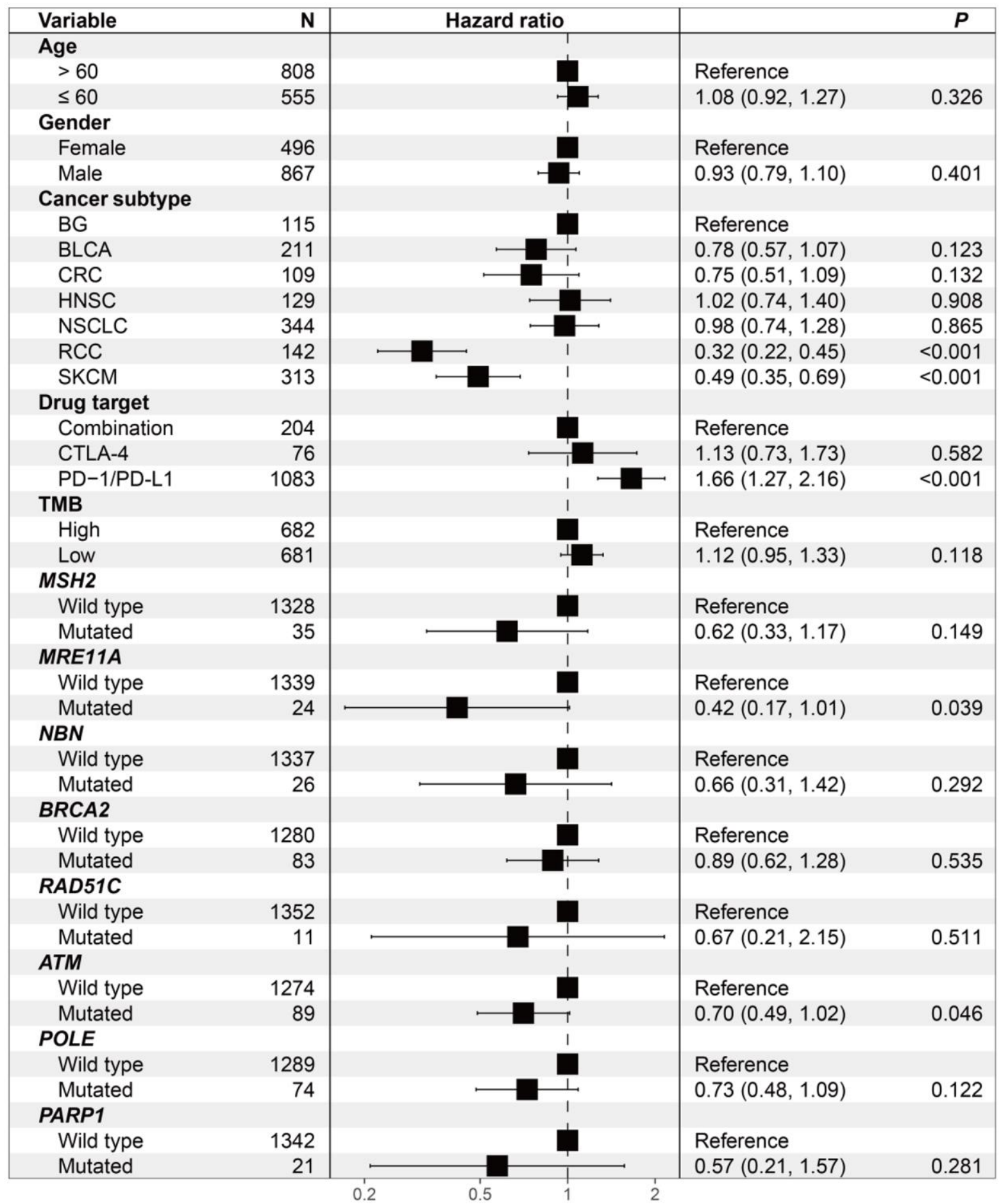

Supplementary Figure 1. Multivariate Cox regression model was conducted with clinical confounding factors and identified 8 DDR genes mutations taken into consideration in the MSKCC cohort. 

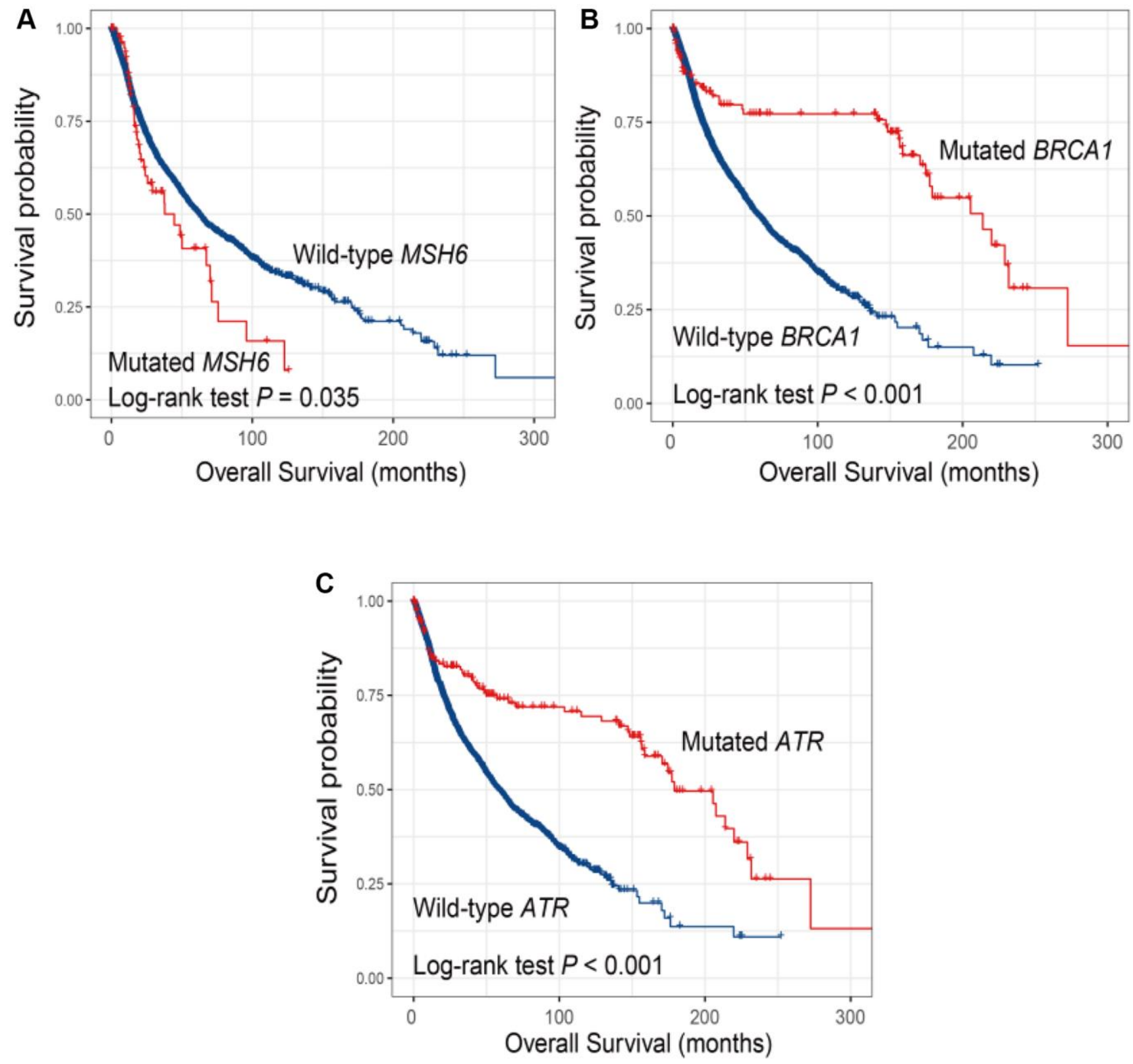

Supplementary Figure 2. Kaplan-Meier survival curves of mutations in 3 DDR genes in the TCGA cohort. Survival curves representation of mutations in (A) MSH6, (B) BRCA1, and (C) ATR. 


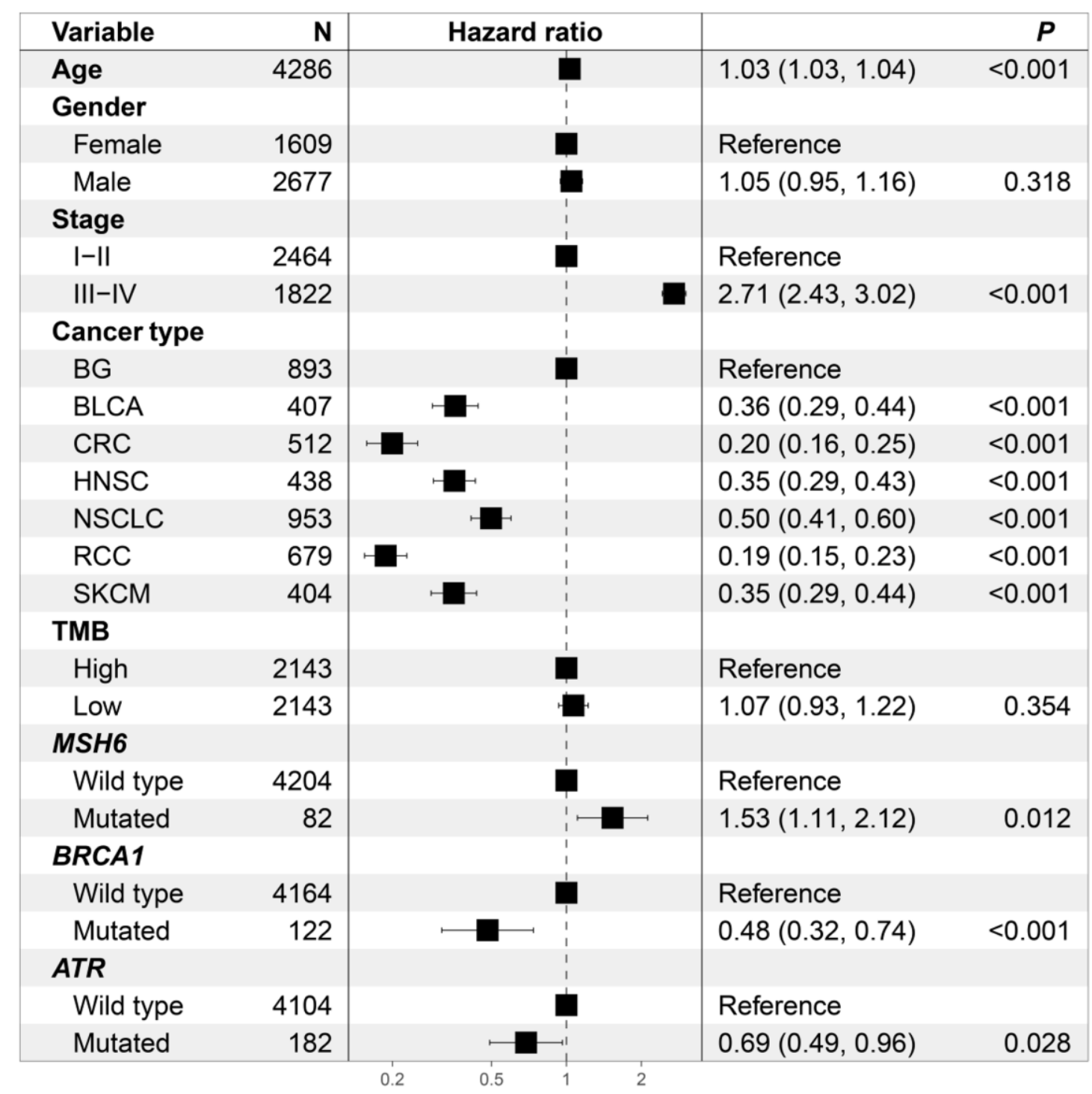

Supplementary Figure 3. Multivariate Cox regression model was conducted with clinical confounding factors and identified 3 DDR genes mutations taken into consideration in the TCGA cohort. 


\section{Supplementary Tables}

Please browse Full Text version to see the data of Supplementary Table 1.

Supplementary Table 1. Clinical characteristics of included 1363 patients treated with immunotherapy.

Supplementary Table 2. Association of DDR mutations with prognosis in distinct conditions in $\mathbf{2}$ cohorts.

\begin{tabular}{lcccccc}
\hline \multirow{2}{*}{ Variables } & \multicolumn{3}{c}{ MSKCC cohort } & \multicolumn{3}{c}{ TCGA cohort } \\
\cline { 2 - 6 } Age & HR $^{*}$ & $\mathbf{9 5 \%}$ CI & $\boldsymbol{P ~ v a l u e}^{*}$ & HR $^{*}$ & $\mathbf{9 5 \%}$ CI & $\boldsymbol{P ~ v a l u e}^{*}$ \\
$\leq 60$ & & & & & & \\
$>60$ & 0.82 & $0.61-1.11$ & 0.198 & 0.71 & $0.57-0.87$ & $\mathbf{0 . 0 0 2}$ \\
Gender & 0.63 & $0.50-0.81$ & $<\mathbf{0 . 0 0 1}$ & 0.9 & $0.78-1.04$ & 0.268 \\
Male & & & & & & 0.069 \\
Female & 0.7 & $0.55-0.89$ & $\mathbf{0 . 0 0 3}$ & 0.87 & $0.75-1.00$ & $\mathbf{0 . 0 0 3}$ \\
TMB & 0.73 & $0.54-0.99$ & $\mathbf{0 . 0 4 4}$ & 0.74 & $0.61-0.90$ & 0.238 \\
Low & & & & & $0.69-1.09$ & $\mathbf{0 . 0 0 7}$ \\
High & 1.17 & $0.88-1.56$ & 0.276 & 0.87 & $0.72-0.95$ & \\
Drug target & 0.56 & $0.45-0.70$ & $<\mathbf{0 . 0 0 1}$ & 0.83 & & \\
PD-1/PD-L1 & & & & & & \\
CTLA-4 & 0.72 & $0.59-0.89$ & $\mathbf{0 . 0 0 2}$ & & & \\
Combined & 0.54 & $0.22-1.35$ & 0.198 & & & \\
\hline
\end{tabular}

${ }^{*} \mathrm{HR}$ and $P$ Values were obtained via multivariate Cox model adjusted confounding factors.

Supplementary Table 3. Mutations of single DDR gene association with survival interval in $\mathbf{2}$ cohorts.

\begin{tabular}{lcc}
\hline & MSKCC cohort & TCGA cohort \\
\cline { 2 - 3 } & Kaplan-Meier $\boldsymbol{P}$ value & Kaplan-Meier $\boldsymbol{P}$ value \\
\hline MLH1 & 0.986 & 0.59 \\
MSH2 & 0.027 & 0.091 \\
MSH6 & 0.085 & 0.035 \\
PMS1 & 0.872 & 0.213 \\
PMS2 & 0.845 & 0.923 \\
ERCC2 & 0.213 & 0.312 \\
ERCC3 & 0.126 & 0.285 \\
ERCC4 & 0.108 & 0.129 \\
ERCC5 & 0.127 & 0.099 \\
BRCA1 & 0.545 & $<0.001$ \\
MRE11A & 0.013 & 0.413 \\
NBN & 0.028 & 0.716 \\
RAD50 & 0.055 & 0.941 \\
RAD51 & 0.224 & 0.644 \\
RAD51B & 0.078 & 0.645
\end{tabular}




\begin{tabular}{lcc} 
RAD51D & 0.412 & 0.535 \\
RAD52 & 0.955 & 0.346 \\
RAD54L & 0.145 & 0.481 \\
BRCA2 & 0.024 & 0.634 \\
BRIP1 & 0.058 & 0.616 \\
FANCA & 0.064 & 0.382 \\
FANCC & 0.206 & 0.557 \\
PALB2 & 0.368 & 0.945 \\
RAD51C & 0.035 & 0.654 \\
BLM & 0.493 & 0.846 \\
ATM & 0.006 & 0.358 \\
ATR & 0.188 & $<0.001$ \\
CHEK1 & 0.456 & 0.756 \\
CHEK2 & 0.635 & 0.978 \\
MDC1 & 0.213 & 0.018 \\
POLE & 0.023 & 0.335 \\
MUTYH & 0.297 & 0.145 \\
PARP1 & 0.017 & 0.923 \\
RECQL4 & 0.334 & 0.112 \\
\hline
\end{tabular}

Supplementary Table 4. DDR-related genes and pathways.

\begin{tabular}{ll}
\hline Genes + A2:B36 & Pathways \\
\hline MLH1 & Mismatch repair (MMR) \\
MSH2 & Mismatch repair (MMR) \\
MSH6 & Mismatch repair (MMR) \\
PMS1 & Mismatch repair (MMR) \\
PMS2 & Mismatch repair (MMR) \\
ERCC2 & Nucleotide excision repair (NER) \\
ERCC3 & Nucleotide excision repair (NER) \\
ERCC4 & Nucleotide excision repair (NER) \\
ERCC5 & Nucleotide excision repair (NER) \\
BRCA1 & Homologous recombination (HR) \\
MRE11A & Homologous recombination (HR) \\
NBN & Homologous recombination (HR) \\
RAD50 & Homologous recombination (HR) \\
RAD51 & Homologous recombination (HR) \\
RAD51B & Homologous recombination (HR) \\
RAD51D & Homologous recombination (HR) \\
RAD52 & Homologous recombination (HR) \\
RAD54L & Homologous recombination (HR) \\
BRCA2 & Fanconi anemia (FA) \\
BRIP1 & Fanconi anemia (FA) \\
FANCA & Fanconi anemia (FA) \\
\hline
\end{tabular}


FANCC

PALB2

RAD51C

BLM

ATM

ATR

CHEK1

CHEK2

MDC1

POLE

MUTYH

PARP1

RECQL4
Fanconi anemia (FA)

Fanconi anemia (FA)

Fanconi anemia (FA)

Fanconi anemia (FA)

Checkpoint

Checkpoint

Checkpoint

Checkpoint

Checkpoint

Others

Others

Others

Others 